\title{
Brecha Digital por Estatus Socio-Económico en la Localidad de Ciudad Bolívar de Bogotá (Colombia)
}

\author{
Luis F. Pedraza, Danilo A. López y Octavio J. Salcedo \\ Universidad Distrital "Francisco José de Caldas", Carrera 7 No. 40 - 53, Bogotá-Colombia. \\ (e-mail: \{lfpedrazam, dalopezs, ojsalcedop\}@udistrital.edu.co)
}

Recibido Mar. 16, 2012; Aceptado May. 02, 2012; Versión final recibida Jul. 11, 2012

\begin{abstract}
Resumen
En este artículo se reporta un análisis de la incidencia de las Tecnologías de la Información y la Comunicación (TIC) en especial para el servicio de Internet en la localidad de Ciudad Bolívar en Bogotá-Colombia, que es uno de los sectores con mayores índices de pobreza en la ciudad. El principal objetivo del estudio fue recopilar la información pertinente para identificar las características de la localidad en cuanto a tecnología y principales necesidades que se puedan suplir o mejorar a través de las TIC. Para caracterizar adecuadamente las necesidades de la población, se realizan tres tipos de encuestas: hogar, entorno y empresarial. Luego, se presentan los resultados estadísticos de este estudio, teniendo en cuenta el error permitido y el nivel de confianza estimado.
\end{abstract}

Palabras clave: tecnologías de la información y la comunicación, estadística paramétrica, brecha digital, alfabetización digital

\section{Digital Gap by Social and Economic Status in the Area of Ciudad Bolívar in Bogotá (Colombia)}

\begin{abstract}
In this paper the analysis of the impact of Information and Communication Technology (ICT) especially for the Internet service in the area of Ciudad Bolivar in Bogota-Colombia, one of the sectors with the highest poverty rates in the city. The main objective of the study is to gather relevant information to identify the characteristics of the area in terms of technology and basic needs that can be supplied or improved through ICT. To properly characterize the needs of the population, three kinds of surveys were done: household, environment and enterprise. Then, the statistical results of this study are presented, taking into account the permissible error and the estimated confidence level.
\end{abstract}

Keywords: information and communication technology, parametric statistics, digital gap, digital literacy 


\section{INTRODUCCIÓN}

El nuevo contexto de la Sociedad de la Información y el desarrollo de las Tecnologías de la Información y la Comunicación inciden en los ámbitos económico, político, social y cultural, lo cual se ha apreciado en diferentes estudios de brecha digital (ALADI, 2003; Rectoría de Telecomunicaciones, 2010; Serrano et al., 2003; Chaparro, 2007; Singh, 2010). Para referirse al empleo de las TIC, se usan conceptos como: Acceso, el cual se refiere a la forma de acercarse a las TIC. Uso, se entiende como el ejercicio o práctica habitual y continuada de un artefacto tecnológico. Apropiación, puede definirse como un ejercicio interpretativo y el desarrollo de unas prácticas reflexivas (Crovi, 2007).

En este trabajo se ofrece una panorámica general de la situación actual de la comunidad de Ciudad Bolívar, que es una localidad que posee el $27 \%$ del área de la Ciudad de Bogotá, es la cuarta localidad en número de habitantes en la ciudad y predomina la clase socioeconómica-baja. Ciudad Bolívar es afectada por los altos índices de desempleo y de empleo informal que son medidos en el estudio de brecha digital que se presenta en este trabajo, donde la brecha digital puede definirse como la distancia "tecnológica" entre individuos, familias, empresas, grupos de interés, países y áreas geográficas en sus oportunidades en el acceso a la información y a las tecnologías de la comunicación y en el uso de Internet para un amplio rango de actividades. Por tanto, la transformación del mercado laboral constituye un exponente claro de los cambios propiciados por la denominada economía digital, la cual es definida en (Australian Government, 2009) como la red global de actividades económicas y sociales que son realizadas a través de las TIC, tales como la Internet, la telefonía móvil y las redes de sensores. Por un lado, emergen nuevos empleos que requieren el dominio de estas tecnologías, por ejemplo en la localidad de Ciudad Bolívar el $73 \%$ del área es rural, lo que podría generar nuevos empleos en la comercialización de productos agrícolas a través de las TIC. Por otro lado, surge una nueva forma de buscar empleo a través de Internet. Las respuestas a las nuevas exigencias que plantea la Sociedad de la Información, entendiendo a esta como una sociedad caracterizada por un modo de ser comunicacional que atraviesa todas las actividades (industria, entretenimiento, educación, organización, servicios, comercio, etc.) (Crovi, 2002), pueden ser directa o indirectamente consideradas estrategias de generación de empleo (Begoña e Ixone, 2002) y una herramienta en los empleos que requieren un alto nivel tecnológico (García, 2008).

Todos los ámbitos, económicos, sociales y culturales, son partícipes de los efectos del desarrollo de las TIC. La innovación y posterior implantación de las nuevas tecnologías constituye un factor estratégico para mejorar el acceso a la educación (Díaz y García, 2011), la competitividad empresarial, impulsar el crecimiento económico y lograr una mayor creación de empleo (Vesisenaho, 2010). De todos los elementos que integran las TIC, sin duda el más poderoso y revolucionario es Internet, que abre las puertas de una nueva era, en la que se ubica la actual Sociedad de la Información (Vidas y Knezevic, 2011). Internet es una fusión entre la comunicación y el entretenimiento a distancia. Estas propiedades de Internet, entre otras, permiten que surjan comunidades en línea y mundos virtuales (Kokswijk, 2010; Jina et al., 1997).

En la actual y global sociedad de la información, existe una correlación directa entre el acceso a las TIC y el desarrollo socio-económico, y las TIC no son la consecuencia del desarrollo, sino más bien una condición previa necesaria para el desarrollo. Aunque las nuevas tecnologías son parte de las necesidades para el desarrollo, los principales "ingredientes" de desarrollo son las propias personas, quienes deben establecer sus objetivos de desarrollo para sus propias comunidades (Nampijja, 2010). Esta es la meta a establecer a partir del estudio de brecha digital que se presenta a continuación; aunque la comunidad académica de la Facultad Tecnológica de la Universidad Distrital ubicada en esta localidad, ya ha desarrollado algunas aplicaciones TIC para sectores más desfavorecidos de la Localidad de Ciudad Bolívar (Pedraza et al., 2010; Rodríguez et al. 2010; Pedraza et al. 2011). Por tanto, este trabajo, aporta una perspectiva de la comunidad de Ciudad Bolívar, que servirá de orientación para el desarrollo del convenio Ciudad Bolívar Localidad Digital, convenio en el que participan entidades como la Empresa de Telecomunicaciones de Bogotá, el Ministerio de TIC, la Alcaldía Mayor de Bogotá mediante sus diferentes secretarias, la Alcaldía Local de Ciudad Bolívar y la Universidad Distrital. Con este 
convenio se busca el desarrollo de una estrategia orientada a alcanzar un amplio acceso a los servicios de TIC y su apropiación en la localidad, lo cual permitiría vislumbrar el potencial de las TIC en el mejoramiento de la calidad de vida de este tipo de comunidades.

\section{METODOLOGÍA}

El desarrollo de esta investigación se basa en el método científico, por lo que la información se recopila a través de encuestas, las encuestas son de tipo exploratorio y el tipo de muestreo es estratificado, debido a la homogeneidad socioeconómica de la población. Las encuestas se realizaron entre los meses de Mayo y Junio de 2011 por 100 estudiantes y docentes de la Universidad Distrital, para el desarrollo de las encuestas se tuvo en cuenta la cantidad de barrios, los cuales conforman las Unidades de Planeamiento Zonal (UPZ) y la cantidad de encuestas a realizar por UPZ para asegurar una muestra adecuada. Por tanto, se hace la relación entre el número total de encuestas y el número de barrios por UPZ para calcular la cantidad de encuestas por barrio a ser aplicadas. Para una población de 616455 habitantes y 5089 microempresas en la Localidad de Ciudad Bolívar, se usa la ecuación 1, para determinar el número de encuestas:

$\mathrm{n}=\frac{\mathrm{Z}^{2} p q \mathrm{~N}}{N \mathrm{E}^{2}+\mathrm{Z}^{2} p q}$

En donde, $n$ es el tamaño de la muestra, $Z$ es el nivel de confianza estimado, $p$ es la variabilidad positiva, $q$ es la variabilidad negativa, $N$ es el tamaño de la población y $E$ el error permitido.

Para una distribución de tipo normal, con un intervalo de confianza del $97 \% Z_{0,97}=2,17$, y los siguientes datos para encuestas de hogar: $E=1,1 \%, p=q=0,5, N=616455$, usando la ecuación 1 , se tiene un $n=9578$ encuestas, que se decide aproximar a 10000 encuestas de hogar. Para determinar el número de encuestas empresariales se usa un $E=3,1 \%, p=q=0,5, N=5089$, reemplazando en la ecuación $1, n=987$ encuestas que se aproximan a 1000. Este tamaño de muestra garantiza una alta probabilidad de poder caracterizar a la población con óptimos índices de confianza y asumir una distribución de tipo normal que caracteriza las muestras mayores a 30 unidades (Venegas y Rojas, 2009).

\section{Clasificación de encuestas}

Para garantizar una caracterización adecuada en los diferentes escenarios de la comunidad de Ciudad Bolívar, se desarrollan las siguientes clases de encuestas, en base a las recomendaciones de medición de brecha digital de la Unión Internacional de Telecomunicaciones (UIT) y de la Comisión Económica para América Latina y el Caribe (CEPAL), que pueden ser consultadas en (UIT, 2010; Schulz y Olaya, 2005):

Encuesta medición de necesidades básicas en el hogar: Este tipo de encuesta va enfocado a caracterizar a las personas que habitan en el sector y que son protagonistas del desarrollo de su localidad, su problemática y sus necesidades personales y familiares. La distribución de este tipo de encuestas parte de la información mostrada en la Fig. 1.

Encuesta Medición de Sector productivo: Esta encuesta va directamente relacionada con la aplicación de las TIC, en especial del servicio de internet, en las Pequeñas y Medianas Empresas (PYMES) de la localidad y de la medición de su impacto, que permita establecer si existe la necesidad de su utilización y del posible mejoramiento en el desarrollo del sector productivo. Como no existe una información de la distribución de las PYMES en la localidad, se escogió relacionarlas directamente con la distribución de la población por UPZ.

Encuesta de Evaluación de necesidades en el entorno para el encuestador: Esta clase de encuesta busca con ayuda de las personas del sector determinar las necesidades que se plasman en los lugares públicos y comunes, como: carreteras, construcciones, parques, centros de capacitación, centros médicos, entre otros. La distribución de estas encuestas depende de la percepción del encuestador. 
En la Tabla 1 se relacionan la cantidad de encuestas realizadas de hogar, empresarial y entorno, a través de recorridos aleatorios y empleando el método de recolección de entrevistas personales, en donde es usada la escala de medida nominal en el cuestionario desarrollado.

La distribución por edad y sexo de la población de la localidad se observa en la Fig. 1. De aquí se observa que el $48,8 \%$ son hombres y el $51,2 \%$ mujeres. La población para los dos sexos, de niños (5 a 9 años) es de 11,9\%, de adolescentes (10 a 19 años) es de 21,2\%, de adultos (20-64 años) es de $52,3 \%$ y ancianos (>65 años) es de $4,1 \%$. Estos porcentajes se intentan mantener en la muestra.

Tabla 1: Numero de encuestas por UPZ.

\begin{tabular}{|l|c|c|c|c|c|c|}
\hline \multirow{2}{*}{$\begin{array}{c}\text { Nombre de } \\
\text { la UPZ }\end{array}$} & \multicolumn{2}{|c|}{ Hogar } & \multicolumn{2}{c|}{ Empresarial } & \multicolumn{2}{c|}{ Entorno } \\
\cline { 2 - 7 } & Meta & Realizada & Meta & Realizada & Meta & Realizada \\
\hline Jerusalén & 1601 & 1620 & 160,1 & 158 & 35 & 18 \\
\hline San Francisco & 1237 & 1175 & 123,7 & 91 & 27 & 12 \\
\hline Arborizadora & 986 & 1099 & 98,6 & 122 & 25 & 18 \\
\hline Lucero & 2639 & 2721 & 263,9 & 278 & 77 & 66 \\
\hline Perdomo & 2659 & 3022 & 265,6 & 281 & 70 & 47 \\
\hline El Tesoro & 773 & 775 & 77,3 & 75 & 50 & 18 \\
\hline Monte Blanco & 84 & 103 & 8,4 & 7 & 9 & 7 \\
\hline Mochuelo & 24 & 68 & 2,4 & 10 & 7 & 5 \\
\hline Total & 10000 & 10583 & 1000 & 1022 & 300 & 191 \\
\hline
\end{tabular}

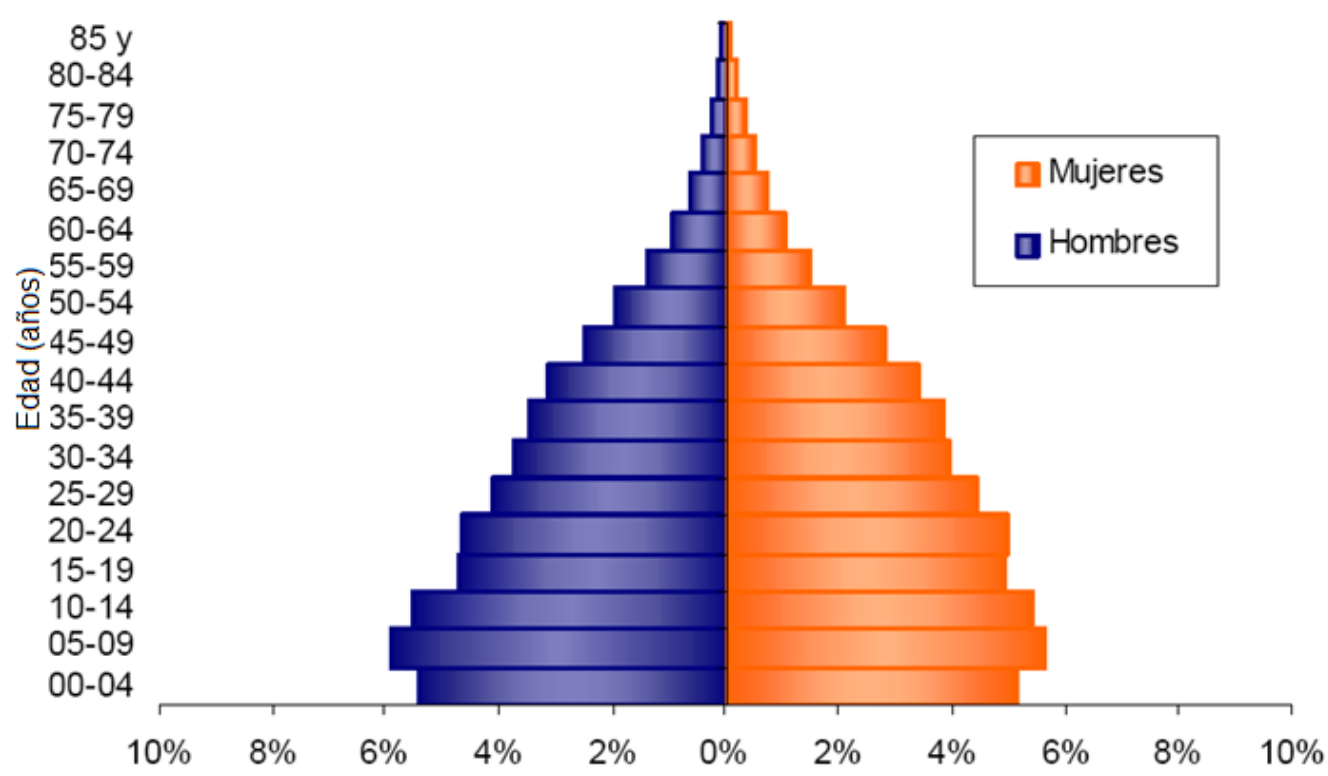

Fig. 1: Distribución porcentual de la población por sexo y edad en grupos quinquenales.

\section{RESULTADOS}

Para esta investigación, en las encuestas descritas anteriormente, se uso la estadística inferencial en base a la estimación por intervalos de confianza (Nolberto y Ponce, 2008; Matilla y Chalmeta, 2007), en el software Minitab.

\section{Análisis hogar}

Los resultados de la población encuestada que habita en Ciudad Bolívar corresponden a:

El 95\% de los habitantes de Ciudad Bolívar pertenece a los estratos 1 y 2 (estratos socioeconómicos bajos).

La cantidad promedio de habitantes por hogar es cercana a 5. 
En su mayoría la ocupación de los encuestados es de estudiantes, seguidos por empleados e independientes.

El análisis del acceso a servicios TIC, por parte de la comunidad encuestada se resume a continuación:

El $88 \%$ cuenta con el servicio de radiodifusión.

El $96 \%$ posee el servicio de televisión.

El porcentaje de penetración de línea fija en la localidad es del 16,1\%.

El porcentaje de penetración de línea móvil es del $84 \%$.

El porcentaje de penetración de computadores es del 6,8\%.

El porcentaje de penetración de computadores sin Internet es del 3\%.

El porcentaje de penetración de computadores con Internet es del 3,8\%.

El $89 \%$ de los encuestados plantean que los medios de comunicación no mejorarían el bienestar en su núcleo familiar.

El $81 \%$ de la población no tiene ningún tipo de conexión a Internet desde su hogar y el $16 \%$ accede por banda ancha fija.

Con respecto al uso de las TIC, se resalta:

El $33 \%$ de la población nunca ha utilizado un computador y el $46 \%$ lo ha utilizado en el último mes de la encuesta que fue en Junio de 2011.

El $35 \%$ nunca ha navegado en Internet y el $44 \%$ lo ha hecho en el último mes.

El lugar más frecuente de acceso a Internet en la localidad es desde un café Internet.

El principal objetivo de navegación en internet es realizar las tareas y consultar el correo electrónico.

De la apropiación de las TIC en la comunidad se puede destacar:

Para el $12 \%$ de los encuestados el Internet no es útil, mientras que para el $39 \%$ es muy útil.

El $40 \%$ de la población utiliza internet con periodicidad que oscila entre 1 y 5 días.

El $81 \%$ de la población está interesada en capacitarse en temas de Internet, la principal temática de interés para capacitación es la forma de navegación en Internet.

Ahora se plantea una hipótesis para analizar a partir de la estadística paramétrica el nivel de asociación entre la edad y el uso de internet de la población en la localidad, como se muestra en la Tabla 2.

Tabla 2: Uso de internet por edad en la población de la localidad.

\begin{tabular}{|c|c|c|c|c|c|c|}
\hline \multirow{3}{*}{ Población } & \multirow{2}{*}{ Internet } & \multicolumn{4}{|c|}{ Edad } & \multirow{2}{*}{ Total } \\
\cline { 3 - 7 } & & Niños & Adolescentes & Adultos & Ancianos & \\
\cline { 2 - 7 } & Usa & 940 & 1875 & 2498 & 106 & 5419 \\
\cline { 2 - 7 } & No usa & 541 & 665 & 3429 & 529 & 5164 \\
\hline \multicolumn{2}{|c|}{ Total } & 1481 & 2540 & 5927 & 635 & 10583 \\
\hline
\end{tabular}

Para este caso las hipótesis son:

$\mathrm{H}_{\text {int: }}$ : Existe relación entre la edad y el uso de internet.

$\mathrm{H}_{0}$ : El uso de internet es independiente de la edad.

Ahora se contrasta la hipótesis haciendo uso de los siguientes valores estadísticos:

Si $X_{\text {cal }}^{2} \geq X_{\text {crit }}^{2}$, se rechaza la $\mathrm{H}_{0}$.

Si $X^{2}$ cal $<X^{2}$ crit, se acepta la $\mathrm{H}_{0}$.

Donde,

$\mathrm{X}_{\mathrm{cal}}^{2}=\sum \frac{(\rho-\mu)^{2}}{\mu^{k}}$

En la ecuación 2, $\rho$ es la frecuencia observada en la celda y $\mu$ es la media. Por tanto, para los valores de la Tabla 2 se tiene $X_{\text {cal }}^{2} \approx 7172$. Para un nivel de confianza del $97 \%$ y un grado de 
libertad $(g /)=3$, se tiene $X^{2}$ crit $=7,81$, entonces $X^{2}{ }_{c a l} \geq X^{2}$ crit, de acá se indica que hay una relación entre la edad y el uso de internet.

En la Tabla 3 se presenta la relación entre el empleo y el uso de las TIC en la población encuestada de la localidad en edad de laborar.

Tabla 3: Asociación entre el uso de internet y empleo en la población de la localidad.

\begin{tabular}{|c|c|c|c|c|}
\hline \multirow{3}{*}{ Población } & \multirow{2}{*}{ Internet } & \multicolumn{2}{|c|}{ Empleado } & \multirow{2}{*}{ Total } \\
\cline { 2 - 5 } & & Si & No & 3138 \\
\cline { 2 - 5 } & Usa & 2625 & 513 & 3999 \\
\cline { 2 - 5 } & No usa & 3051 & 948 & 7137 \\
\hline
\end{tabular}

Las hipótesis a analizar son:

$\mathrm{H}_{\text {int }}$ : Existe relación entre el empleo y el uso de internet.

$\mathrm{H}_{0}$ : El estar empleado es independiente del uso de internet.

Reemplazando en la ecuación 2 , se obtiene $X_{\text {cal }}^{2} \approx 2527$ y partiendo de un nivel de confianza del $97 \%$ y $g l=1$, se tiene $X^{2}$ crit $=3,84$, entonces $X^{2}{ }_{c a l} \geq X^{2}{ }_{\text {crit }}$, de acá se indica que hay una relación entre el empleo y el uso de internet.

\section{Análisis empresarial}

De las 1022 encuestas realizadas en las UPZ de la localidad de Ciudad Bolívar, en la Tabla 4 se presentan las principales áreas a las que se dedica el sector productivo.

De la Tabla 4 se puede inferir que la gran mayoría del sector empresarial de la localidad se dedica al comercio, seguido por los servicios y con un bajo porcentaje dedicado a la producción industrial. Existen diversos tipos de sector productivo como: tiendas, alimentos, apuestas, servicios de comunicaciones, dulcerías, heladerías, fabricas de bolsas, costuras, café Internet, panaderías, peluquerías, producción de carbón, reciclaje, restaurantes, cacharrerías, misceláneas, cigarrerías, venta de uniformes.

Tabla 4: Áreas a las que se dedica el sector productivo de la localidad.

\begin{tabular}{|l|c|}
\hline Sector Productivo & $\begin{array}{c}\text { Porcentaje } \\
\text { de dedicación }\end{array}$ \\
\hline Agrícola & 0,19 \\
\hline Comercial & 73,29 \\
\hline Industrial & 2,66 \\
\hline Manufacturas & 0,19 \\
\hline Servicios & 20,82 \\
\hline Otros & 2,85 \\
\hline
\end{tabular}

La mayor cantidad de empresas de la localidad cuentan con menos de 10 trabajadores.

Solamente el $14 \%$ de las empresas de la localidad cuentan con computadores.

El uso que las personas le dan al computador en la empresa es muy bajo y se discrimina para actividades como las mostradas en la Tabla 5.

Solamente el $11,22 \%$ de los encuestados tienen Internet en su empresa.

La gran mayoría de las empresas que tienen el servicio de Internet lo hacen a través de ADSL (Línea de Abonado Digital Asimétrica).

La mayor cantidad de usuarios de servicios de Internet en la localidad posee Banda Ancha con un enlace superior a $1 \mathrm{Mbps}$. 
Tabla 5: Actividades para las que los encuestados usan el computador en la empresa.

\begin{tabular}{|l|c|}
\hline \multicolumn{1}{|c|}{ Actividad } & $\begin{array}{c}\text { Porcentaje } \\
\text { de uso }\end{array}$ \\
\hline Contabilidad & 3,90 \\
\hline Gestión administrativa & 2,66 \\
\hline Ventas & 3,9 \\
\hline Producción & 2,19 \\
\hline Capacitación & 0,29 \\
\hline Comunicación & 2,47 \\
\hline Búsqueda de información & 2,38 \\
\hline Investigación & 1,14 \\
\hline $\begin{array}{l}\text { Otro (Internet, Diseño gráfico, } \\
\text { Diseños, Cabinas) }\end{array}$ & 15,59 \\
\hline
\end{tabular}

El uso de Internet en las empresas es variado, especialmente apoya a las ventas, Cerca del $58 \%$ de las empresas consideran que Internet puede mejorar la productividad de la misma. Alrededor del $54 \%$ de las empresas no cuenta con personas capacitadas en ofimática en su organización. El $16 \%$ de las empresas cuenta con personal capacitado en mantenimiento de computadores.

A continuación se analiza si existe relación entre el nivel de ganancias económicas y el uso de internet en las microempresas de la localidad, como se puede observar en la Tabla 6.

Tabla 6: Relación entre el uso de internet y el nivel de ganancias en las microempresas de la localidad.

\begin{tabular}{|c|c|c|c|c|c|}
\hline \multirow{4}{*}{ Empresas } & \multirow[b]{2}{*}{ Internet } & \multicolumn{3}{|c|}{ Ganancias Mensuales } & \multirow[b]{2}{*}{ Total } \\
\hline & & $\begin{array}{c}\text { Bajas } \\
(<250 \cup S D)\end{array}$ & $\begin{array}{c}\text { Medias } \\
(>250 \text { y d y } \\
<500 \text { SD })\end{array}$ & $\begin{array}{c}\text { Altas } \\
(>500 U S D)\end{array}$ & \\
\hline & Usa & 11 & 29 & 75 & 115 \\
\hline & No usa & 514 & 345 & 48 & 907 \\
\hline \multicolumn{2}{|c|}{ Total } & 525 & 374 & 123 & 1022 \\
\hline
\end{tabular}

Las hipótesis planteadas son:

$\mathrm{H}_{\text {int }}$ : Existe relación entre las ganancias y el uso de internet.

$\mathrm{H}_{0}$ : El uso de internet es independiente a las ganancias.

Haciendo uso de la ecuación 2 , se tiene $X_{\text {cal }}^{2} \approx 425$. Para un nivel de confianza del $97 \%$ y $g l=2$, resulta $X^{2}$ crit $=5,99$, entonces $X^{2}{ }_{c a l} \geq X^{2}$ crit, con lo que se observa que hay una asociación entre las ganancias y el uso de internet en las PYMES de la localidad.

\section{Análisis entorno}

Para esta fase se realizaron 191 encuestas en los diferentes barrios de la localidad, entre lo que se destaca lo siguiente:

Alrededor del $84 \%$ de las calles principales de los barrios, se encuentran pavimentadas y el $48,3 \%$ de las calles secundarias son destapadas.

Los materiales predominantes de los sitios construidos son bloque, ladrillo, piedra y madera pulida. Solamente el $3 \%$ es material de reciclaje.

El $61,24 \%$ de los barrios cuenta con viviendas ubicadas en zonas de alto riesgo natural, como deslizamientos e inundaciones.

El $20 \%$ de los barrios no cuenta con colegios; mientras un $32 \%$ cuenta con más de dos colegios.

El $75 \%$ de los barrios cuenta con uno o más salones comunales.

El $71 \%$ de los barrios posee como mínimo un comedor comunitario.

El $62 \%$ de los barrios no tiene un centro médico. 
El $79 \%$ de los barrios cuenta con más de un café Internet.

El $83 \%$ de los barrios no tiene zona industrial; mientras en el $57 \%$ hay zonas comerciales.

En el $14,6 \%$ de los barrios hay centros de formación para el trabajo.

En el $89 \%$ de los barrios el costo de la hora para el acceso a Internet es menor o igual a USD0,5.

El costo de un minuto para llamar a celular en los comercios de los barrios de la localidad oscila entre 8 y 11 centavos de dólar.

\section{CONCLUSIONES}

A partir de este estudio, es notoria una gran brecha digital en la comunidad de Ciudad Bolívar, lo que no es más que una manifestación de las inequidades económicas, políticas y sociales que sufre esta comunidad.

Aunque el uso de internet no es una generalidad en la localidad, es visible un uso mayor de internet por parte de las personas jóvenes y las microempresas que poseen mayores ganancias económicas. A su vez, las personas en edad de laborar que usan internet están empleadas en un porcentaje mayor al $7 \%$, en comparación con las que no lo usan.

La consolidación de este estudio inicial para la comunidad de Ciudad Bolívar descrito anteriormente, es de vital importancia para establecer la directriz del convenio Ciudad Bolívar Localidad Digital, el cual en una primera instancia busca aumentar la alfabetización digital de la población, a través de la capacitación de las personas en centros dotados con infraestructura para el acceso a TIC (especialmente internet), llamados portales interactivos.

Un alto porcentaje de la población de la localidad considera que los medios de comunicación no mejorarían el bienestar de su familia, pues en su mayoría los contemplan como una herramienta para el entretenimiento. Adicionalmente, como consecuencia de la baja penetración de internet, la comunidad no conoce a fondo los beneficios de las TIC.

Es relevante que la comunidad académica, como docentes y estudiantes de la Facultad Tecnológica de la Universidad Distrital, se integren a la solución de necesidades que afectan a las personas de esta localidad, a través de herramientas como el desarrollo de aplicaciones TIC sociales, que conlleven a mejorar la calidad de vida de los individuos de este sector. Esto en concordancia con el Plan Vive Digital liderado por el Ministerio de las TIC que busca, entre otros, la masificación de Internet a nivel nacional.

El establecimiento en la localidad de una adecuada política educativa en TIC puede ser un instrumento para combatir la pobreza del sector; siempre y cuando las políticas económicas y sociales actúen en la misma dirección de dicha política educativa.

Las TIC y la educación son herramientas fundamentales para el acceso de la comunidad de Ciudad Bolívar a la sociedad de la información; sin embargo es indispensable la generación de un conocimiento que permita el desarrollo de los diferentes integrantes de esta localidad.

\section{REFERENCIAS}

ALADI, La Brecha Digital y sus Repercusiones en los Países Miembros de la ALADI, http://www.aladi.org, (2003).

Australian Government, Australia's Digital Economy: Future Directions, http://www.dbcde.gov.au, Australian (2009).

Begoña, E. y A. Ixone, El Desarrollo de las Tecnologías de la Información y La Comunicación: Un Nuevo Reto para el Mercado de Trabajo, Revista Electrónica de Geografía y Ciencias Sociales, http://www.ub.edu/geocrit/sn/sn119-74.htm, 6(119), (2002). 
Chaparro, J., La segregación digital en América Latina y el Caribe: reflejo de las inequidades sociales y la dependencia tecnológica. Revista electrónica de geografía y ciencias sociales. http://www.ub.es/geocrit/sn/sn-24523.htm, 11(245), (2007).

Crovi, D., Acceso democrático, uso compartido y apropiación cultural de las TIC, XIX Encuentro Nacional AMIC "Las claves necesarias de una Comunicación para la Democracia", México (2007).

Crovi, D., Sociedad de la información y el conocimiento. Entre el optimismo y la desesperanza, Revista Mexicana de Ciencias Políticas y Sociales: XLV(185), 13-33 (2002).

Díaz, I. y M. García, Más Allá del Paradigma de la Alfabetización: La Adquisición de Cultura Científica como Reto Educativo, Formación Universitaria: 4(2), 3-14 (2011).

García, J., Concentración de sectores intensivos en conocimiento y de alta tecnología: el caso de España, Journal of Technology Management \& Innovation: 3(4) 66-79 (2008).

Jina, J. y Wright, D, Competitive advantage from the Internet, Manufacturing Engineer: (76)3, 124128, (1997).

Kokswijk V., Social Control in Online Society-Advantages of Self-Regulation on the Internet, Cyberworlds (CW), 2010 International Conference on , 239-246, (2010).

Matilla, M. y R. Chalmeta, Metodología para la Implantación de un Sistema de Medición del Rendimiento Empresarial, Información tecnológica: 18(1), 119-126 (2007).

Nampijja, D., The role of ICT in community rural development, Master Thesis, University of Agder, Faculty of Economics and Social Sciences, Mpigi, Uganda (2010).

Nolberto, V. y M. Ponce, Estadística Inferencial Aplicada, $1^{\text {a }}$ edición, 21-44. Universidad Nacional Mayor de San Marcos, Lima, Perú (2008).

Pedraza, L., C. Torres y U. León, Intervención Social: El Compromiso de la Academia con Ciudad Bolívar, Visión Electrónica: 4(2), 136 - 145 (2010).

Pedraza, L., R. Ramírez y O. Hernández, Diseño e implementación de una plataforma piloto de marketing electrónico para el desarrollo de aplicaciones TICs en la comunidad de Ciudad Bolívar, Tecnura: 15(28), 42 - 52 (2011).

Rectoría de Telecomunicaciones, Evaluación de la Brecha Digital en el Uso de los Servicios de Telecomunicaciones en Costa Rica, Costa Rica (2010).

Rodríguez, N., W. Cortes, y L. Pedraza, Optimización Tecnológica en el Colegio Unión Europea de la Localidad de Ciudad Bolívar, Tecnura: 14(26), 49 - 55 (2010).

Serrano, A. y E. Martínez, La Brecha Digital Mitos y Realidades, $1^{\text {a }}$ edición, 93-104. Universidad Autónoma de Baja California, Baja California, México (2003).

Singh, S., Digital Divide in India: Measurement, Determinants and Policy for Addressing the Challenges in Bridging the Digital Divide. International Journal of Innovation in the Digital Economy: 1(2), 1-24 (2010).

Schulz, C., y D. Olaya, Hacia la construcción de un instrumento de medición para la Sociedad de la Información en América Latina y el Caribe: censos, encuestas de hogares y empresas, http://www.eclac.org/ddpe/publicaciones/xml/0/36110/W224.pdf, Naciones Unidas, Santiago de Chile (2005). 
UIT, Partnership para la Medición de las TIC para el Desarrollo, http://www.itu.int/dms_pub/itud/opb/ind/D-IND-ICT_CORE-2010-PDF-S.pdf, Ginebra, Suiza (2010).

Venegas, F. y R. Rojas, Teoría y Práctica del Ordenamiento y Manejo Sustentable del Territorio: Tijuana-Rosarito-Tecate, Baja California, México, Información Tecnológica: 20(3), 73 87 (2009).

Vesisenaho, M., ICT education and computer science education for development - Impact and contextualization, Frontiers in Education Conference (FIE), IEEE 2010, Washington, D.C., USA, 27 al 30 de Octubre (2010).

Vidas, M. y Knezevic, B., The importance of ICT research and development for innovative eBusiness application and information society, MIPRO, 1396-1401, (2011). 